\title{
Discussion to papers of G. M. Pool and R. Turk et al.
}

\author{
Chairman: DR J. Young
}

DR Hardy (G.B.). Just one comment and one question. In order to get a good response from an electrical stimulus applied to the skin you must have an intact reflex arc, very important indeed. So this really excludes certain categories of lesions. May I ask what was the level and degree of completeness of the lesion of the man who was shown on the film and may I ask also whether there were any problems later with adductor spasm and plantarflexion of the feet?

DR TURK (Yugoslavia). We didn't record that the spasms increased with electrical stimulation. Even we could realise that the spasms decreased after the procedures, after finishing electrical stimulation.

DR M. MAURY (France). Just a question. When you stimulate the extensors muscle, quadriceps during some weeks or months, have you observed imbalance of the spasticity with prominence of the spasticity in the extensor?

DR TURK. We have up to now tried only three patients with this extensive programme and all of these patients claimed that they prefer this system because they feel that the spasms are really decreasing but of course when the patients have been home for 3 or 4 months they state that they would like to have electrical stimulation again.

DR YounG. Before we get to the last question, which is the gentleman back there, the moderator would like to say I am always concerned about these sort of techniques because we do have difficulty convincing our people that they are not going to walk and sometimes I think the stimulation technique reinforces these sort of negative things, not that I am discouraging you at all, but I think sometimes the psychological disadvantages overweigh our attempts.

DR G. SMITH (U.S.A.). The method of movement shown between the parallel bars meant that immediately the heel struck, the knee was thrown into severe hyperextension in order to balance. If an orthodist was making a brace for this patient he would prevent recurvation by building in a certain amount of flexion as in the Craig Scott brace. Have you considered using a brace in order to eliminate this? Have you considered that this can only be a short-term measure because otherwise he will damage the ligaments of the knee? 